\title{
RESPONSE OF YIELD AND ITS QUALITY OF SWEET PEPPER PLANT TO CERTAIN BIO-STIMULANTS UNDER TWO TYPES OF SALINITY
}

\author{
Arafa,A.A., Khafagy,M.A., Abo-El Kheer,A.M., and M.F. El-Banna
}

Dept. Agric. Bot., Fac. of Agric., Mansoura Univ., El-Mansoura, Egypt
E-Mail: dr.arafa50@yahoo.com; khafagy@mans.edu.eg; prof.aboelkheer@gmail.com and El-Banna@mans.edu.eg;

\section{ABSTRACT}

Number of fruits and its fresh weight per plant were significantly increased under low salinity level of $\mathrm{NaCl}+\mathrm{CaCl}_{2}$ in the early 4th picking and total yield. While, plants grown under $\mathrm{NaCl}$ salinity did not produced fruits in the early 4th picking. However, increasing salinity levels caused a significant decrease in this respect. In addition, pre-soaking seeds in selected chemicals used at both levels caused a significant increase in sweet pepper fruit number and its fresh weight per plant (early 4th picking and total yield) under non-saline conditions. Moreover, salicylic acid at $75 \mathrm{mg} / \mathrm{L}$ or ascorbic acid at $50 \mathrm{mg} / \mathrm{L}$ was more effective in this respect under non-saline or salinity conditions.

Ascorbic acid as well as total carbohydrates concentrations in sweet pepper fruits were increased significantly with increasing salinity levels. In addition, ascorbic acid and total carbohydrates concentrations were greatly increased in plants growing under $\mathrm{NaCl}$ followed by $\mathrm{NaCl}+\mathrm{CaCl}_{2}$ and $\mathrm{CaCl}_{2}$ as compared to control. On the other hand, pre-soaking seeds in SA, AsA, a-tocopherol and yeast extract at both levels increased ascorbic acid and total carbohydrates concentrations under non-saline or salinity conditions. Furthermore, AsA at both levels or SA at $75 \mathrm{mg} / \mathrm{L}$ was more effective in this respect.

Total soluble solids (TSS \%) were significantly increased under low salinity level (2000 mg/L), thereafter decreased with increasing salinity level to $4000 \mathrm{mg} / \mathrm{L}$. In addition, the great reduction in TSS \% occurred under $\mathrm{NaCl}$ at high salinity levels as compared with the other salinity type. Moreover, pre-soaking seeds in chemicals used at both levels increased TSS \% under non-saline or saline conditions. Furthermore, SA at $75 \mathrm{mg} / \mathrm{L}$ or AsA at $50 \mathrm{mg} / \mathrm{L}$ was more effective in this respect.

Keywords: Salinity, Bio-stimulants, Sweet Pepper

\section{Council for Innovative Research}

Peer Review Research Publishing System

Journal of Advances in Natural Sciences

Vol 2, No. 2

editorjansonline@gmail.com

www.cirworld.com

72 | $P$ a g e

October 08,2014 


\section{INTRODUCTION}

Sweet pepper (Capsicum annuum L.) is among the most important crops for the world human nutrition and its fruits have a good nutritional value in respect to antioxidant compounds, such as vitamin $\mathrm{C}$ and carotenoids (Navarro et al., 2006).

It is a moderately-sensitive to salt stress (Lycoskoufis et al., 2005). It cultivated under open field and greenhouses conditions. In Egypt cultivated area is around 71428.57 Feddan in 2008, yielded 475000 tons (FAO, 2008) ${ }^{\star 1}$. In addition, productions throughout the world are around over 24 million tons every year (Casado-Vela et al., 2007). Soil salinity is one of the major environmental stresses affecting over $20 \%$ of the world's irrigated land (Etehadnia, 2009) and $2.1 \%$ of the dry-land agriculture existing on the globe (Khosravinejad et al., 2009) and extent throughout the world is increasing regularly (Schwabe et al., 2006). It has now become a very serious problem for crop production (Munns and Tester, 2008), particularly in arid and semi-arid regions. However, the intensity of salinity stress varies from place to place. Irrigated land produces one-third of the world's food approximately (Munns, 2002) so its salinization, often due to poor irrigation practices, is particularly critical. Dry land salinity is also an important, and increasing, problem in some areas of the world (Tester and Davenport, 2003).

Therefore, the present investigation was performed to study the effect of different sources of salinity $(\mathrm{NaCl}$, $\mathrm{CaCl}_{2}$ and its combination 1:1) on yield and its quality of sweet pepper plant. Moreover, it was intended to investigate effects of pre-soaking seeds in some materials such as vitamins (ascorbic acid and a-tocopherol, bio-regulator (salicylic acid) and Yeast extract to alleviate the harmful effects of such salinity types.

\section{MATEREIALS AND METHODS}

The experiment was carried out in the glasshouse of the Agricultural Botany Dept., Fac. of Agriculture, Mansoura Univ. during the growing season of 2008, to study the response yield and its quality of sweet pepper to different sources of salinity i.e. $\mathrm{NaCl}, \mathrm{CaCl}_{2}$ and their combination $(1: 1 \mathrm{w} / \mathrm{w})$; and how to minimize its harmful effects through pre-soaking seeds in vitamins (Ascorbic acid or a-tocopherol) or bio-regulators (Salicylic acid) or Yeast extract.

\section{Plant materials}

The seeds of sweet pepper (Capsicum annuum L. cv. Orlando), a hybrid 'California Wonder' used in this investigation were secured from the Gohara Co. Cairo, Egypt.

Chemicals:-

1. Vitamins, ascorbic acid Vit. C (AsA) and $\alpha$-tocopherol Vit. E ( $\alpha$-tocopherol.) were supplied by Sigma Chemicals Co., USA and used at the concentration of 50 or $100 \mathrm{mg} / \mathrm{L}$ each.

2. Bio-regulator, salicylic acid (SA) (2-hydroxybenzoic acid) was obtained from Sigma Chemicals, Co., USA. and initially dissolved in $100 \mu \mathrm{L}$ dimethyl sulfoxide and used at the concentrations of 75 and $150 \mathrm{mg} / \mathrm{L}$,

3. Yeast extract, active dry yeast (Saccharomyces cervisiae) was applied at the concentration of $1000 \mathrm{or} 2000 \mathrm{mg} / \mathrm{L}$.

4. Salts:

4.1. Sodium Chloride ( $\mathrm{NaCl}$ ) from EL-Gomhoria Co., Egypt and was used at the concentrations of 2000 and 4000 $\mathrm{mg} / \mathrm{L}$.

4.2. Calcium Chloride $(\mathrm{CaCl} 2)$ from EL-Gomhoria Co., Egypt and was used at the concentrations of 2000 and $4000 \mathrm{mg} / \mathrm{L}$.

4.3. Their combination, $\mathrm{NaCl}: \mathrm{CaCl} 21: 1(\mathrm{w} / \mathrm{w})$ was used at the concentrations of 2000 and $4000 \mathrm{mg} / \mathrm{L}$.

\footnotetext{
** FAO: Food and Agriculture Organization of the united nation, Statistical agricultural database sector. www.http:// faostat.fao.org/site/567/
} 
Table (1): The Molarity (Mol), Electrical Conductivity (E.C.) and pH values for different nutrient solutions.

\begin{tabular}{|c|c|c|c|c|c|c|c|c|c|}
\hline \multirow{3}{*}{\begin{tabular}{|l|} 
Nutrient \\
solution \\
(N.S.) \\
mg/L
\end{tabular}} & \multirow[b]{3}{*}{ N.S. } & \multicolumn{2}{|c|}{ N.S.+ NaCl } & \multicolumn{2}{|c|}{ N.S.+ $\mathrm{CaCl}_{2}$} & \multicolumn{4}{|c|}{ N.S.+ $\left\{\mathrm{NaCl}+\mathrm{CaCl}_{2}\right\}(1: 1) \mathrm{w} / \mathrm{w}$} \\
\hline & & \multirow{2}{*}{$\begin{array}{l}2000 \\
\mathrm{NaCl}\end{array}$} & \multirow{2}{*}{$\begin{array}{l}4000 \\
\mathrm{NaCl}\end{array}$} & \multirow{2}{*}{$\begin{array}{l}2000 \\
\mathrm{CaCl}_{2}\end{array}$} & \multirow{2}{*}{$\begin{array}{l}4000 \\
\mathrm{CaCl}_{2}\end{array}$} & \multicolumn{2}{|c|}{$2000\left(\mathrm{NaCl}+\mathrm{CaCl}_{2}\right)$} & \multicolumn{2}{|c|}{$4000\left(\mathrm{NaCl}+\mathrm{CaCl}_{2}\right)$} \\
\hline & & & & & & $\begin{array}{l}1000 \\
\mathrm{NaCl}\end{array}$ & $\begin{array}{l}1000 \\
\mathrm{CaCl}_{2}\end{array}$ & $\begin{array}{l}2000 \\
\mathrm{NaCl}\end{array}$ & $\begin{array}{l}2000 \\
\mathrm{CaCl}_{2}\end{array}$ \\
\hline Mol (M) & $\begin{array}{c}0 \\
\text { (Control) }\end{array}$ & $3.4 \times 10^{-2}$ & $6.9 \times 10^{-2}$ & $2.0 \times 10^{-2}$ & $3.6 \times 10^{-2}$ & $1.7 \times 10^{-2}$ & $0.9 \times 10^{-2}$ & $3.4 \times 10^{-2}$ & $2.0 \times 10^{-2}$ \\
\hline Ec dSm ${ }^{-1}$ & 2.00 & 5.42 & 8.42 & 4.59 & 7.60 & \multicolumn{2}{|c|}{5.08} & \multicolumn{2}{|c|}{8.08} \\
\hline $\mathrm{pH}$ & 5.50 & 5.77 & 5.80 & 5.19 & 5.30 & \multicolumn{2}{|c|}{5.45} & \multicolumn{2}{|c|}{5.34} \\
\hline
\end{tabular}

Table (2): Weights (g) of pure substances to be dissolved in 1000 liters of water to give the theoretically ideal concentrations (Cooper, 1979).

\begin{tabular}{|l|l|l|}
\hline Substance & Formula & Weight \\
\hline Potassium dihydrogen Phosphate & $\mathrm{KH}_{2} \mathrm{PO}_{4}$ & 263 \\
\hline Potassium Nitrate & $\mathrm{KNO}_{3}$ & 583 \\
\hline Calcium Nitrate & $\mathrm{Ca}\left(\mathrm{NO}_{3}\right)_{2} \cdot 4 \mathrm{H} 2 \mathrm{O}$ & 1003 \\
\hline Magnesium Sulphate & $\mathrm{MgSO}_{4} \cdot 7 \mathrm{H}_{2} \mathrm{O}$ & 513 \\
\hline EDTA Iron & $\mathrm{CH}_{2} \cdot \mathrm{N}_{\left.\left(\mathrm{CH}_{2} \cdot \mathrm{COO}\right)_{2}\right]_{2} \mathrm{Fe} \mathrm{Na}}^{79.0}$ \\
\hline Manganous Sulphate & $\mathrm{MnSO}_{4} \cdot \mathrm{H}_{2} \mathrm{O}$ & 6.10 \\
\hline Boric Acid & $\mathrm{H}_{3} \mathrm{BO}_{3}$ & 1.70 \\
\hline Copper Sulphate & $\mathrm{CuSO}_{4} \cdot 5 \mathrm{H}_{2} \mathrm{O}$ & 0.39 \\
\hline Ammonium Molybdate & $\left(\mathrm{NH}_{4}\right)_{6} \mathrm{Mo}_{7} \mathrm{O}_{24} \cdot 4 \mathrm{H}_{2} \mathrm{O}$ & 0.37 \\
\hline Zinc Sulphate & $\mathrm{ZnSO}_{4} \cdot 7 \mathrm{H}_{2} \mathrm{O}$ & 0.44 \\
\hline
\end{tabular}

After soaking, the sterilized seeds $(25$ seeds/dish) were placed in glass Petri dishes $(11 \mathrm{~cm})$ with a double layer of Whatman No. 1 filter paper. The dishes were left in an incubator in the dark for seed germination at $25 \pm 2{ }^{0} \mathrm{C}$ and $90 \%$ relative humidity, and then dishes were covered with aluminum foils for darkness. In order to avoid water losses, $5 \mathrm{ml}$ of the nutrient solution were added to Petri dishes, every 5 days. Thiram was added to the solution at a concentration of $2 \%$ $(w / v)$ to control the fungi infection.

Table (3): Composition of yeast extract (according to, Nagodawithana, 1991)

\begin{tabular}{|l|l|}
\hline Constituents & Value (\%) \\
\hline Protein & 47 \\
\hline Carbohydrates & 33 \\
\hline Minerals & 8 \\
\hline Nucleic acids & 8 \\
\hline Lipids & 4 \\
\hline \multicolumn{1}{|c|}{ Approximate composition of vitamins } \\
\hline Vitamins & Value ( $\mathbf{\mu g} / \mathbf{g})$ \\
\hline Cholin & 4000 \\
\hline Niacin & $300-500$ \\
\hline Thiamine $\left(\mathrm{B}_{1}\right)$ & $60-100$ \\
\hline Pantorhenate $\left(\mathrm{B}_{5}\right)$ & 70 \\
\hline Riboflavin $\left(\mathrm{B}_{2}\right)$ & $35-50$ \\
\hline Pyridoxine $\mathrm{HCL}\left(\mathrm{B}_{6}\right)$ & 28 \\
\hline Folic acid & $5-13$ \\
\hline
\end{tabular}




\begin{tabular}{|l|l|l|l|}
\hline \multicolumn{2}{|c|}{ Biotin } & 1.3 \\
\hline Vit. $\mathrm{B}_{12}$ & \multicolumn{2}{|c|}{0.001} \\
\hline \multicolumn{4}{|c|}{ Approximate composition of minerals } \\
\hline Minerals & Value (mg/g) & Minerals & Value ( $\mathbf{g} / \mathbf{g})$ \\
\hline $\mathrm{K}$ & 21 & $\mathrm{Cu}$ & 8.00 \\
\hline $\mathrm{P}$ & 13.50 & $\mathrm{Ni}$ & 3.00 \\
\hline $\mathrm{S}$ & 3.90 & $\mathrm{Sn}$ & 3.00 \\
\hline $\mathrm{Mg}$ & 1.65 & $\mathrm{Cr}$ & 2.20 \\
\hline $\mathrm{Ca}$ & 0.75 & $\mathrm{Mo}$ & 0.40 \\
\hline $\mathrm{Zn}$ & 0.17 & $\mathrm{Se}$ & 0.10 \\
\hline $\mathrm{Na}$ & 0.12 & $\mathrm{Li}$ & 0.17 \\
\hline $\mathrm{Si}$ & 0.03 & $\mathrm{Va}$ & 0.04 \\
\hline $\mathrm{Fe}$ & 0.02 & $\mathrm{Mn}$ & 0.02 \\
\hline
\end{tabular}

The following experiment was carried out in the glasshouse of the Agric. Bot. Dept., Fac. of Agric., Mansoura Univ. during the spring-summer period of 2008 in a glasshouse under conditions of ambient light during winter, spring and early summer, with $10 / 14$ light/dark period at $800-1100 \mu \mathrm{mol} \mathrm{m} \mathrm{m}^{-2}-1$ PPFD, a day/night average temperature cycle of $26 / 15^{\circ} \mathrm{C}$ and $65 \pm 5 \%$ relative humidity.

The focus of the current experiment was to provide fundamental biological understanding and knowledge on sweet pepper plants growing in nutrient film technique (NFT), under different sources of salinity $\mathrm{NaCl}, \mathrm{CaCl}{ }_{2}$ and their combinations $1: 1(\mathrm{w} / \mathrm{w})$; and how to minimizing the harmful effects through pre-soaking seeds in vitamins (Ascorbic acid, a-tocopherol) or bio-regulator (Salicylic acid), or Yeast extract. The seeds of sweet pepper were sown on Jan, 13, 2008. A homogenous sweet pepper seeds were placed in $100 \mathrm{ml}$ beakers and $20 \mathrm{ml}$ of $1 \%$ sodium hypochlorite was added for sterilization. These were left in the solution for 5 min followed by washing under running tap water and ionized water twice. Then divided into 9 sets. The first set was soaked (24hours) in distilled water as control and the remaining sets (8) were separately soaked for $24 \mathrm{~h}$ in aqueous solution of AsA or a-tocopherol at (50 or $100 \mathrm{mg} / \mathrm{L})$ each or SA at (75 or $150 \mathrm{mg} / \mathrm{L})$ or Yeast extract at (1000 or $2000 \mathrm{mg} / \mathrm{L})$. Then germinated in seedling trays (209 eye) containing peat moss and perlite $(1: 1)$ as a rooting medium moistened by nutrient cooper solution (Cooper, 1979). Trays containing the seeds were placed in a glasshouse at $28 \pm 2^{0} \mathrm{C}$ to germinate.

The experimental layout consisted of 7 automatic hydroponic units (groups) (experimental plots). Each hydroponic unit comprised of two plastic channels $(4 \mathrm{~m}$ long * $10 \mathrm{~cm}$ in diameter) placed on one side of the holder $(4 \mathrm{~m}$ length * $1.5 \mathrm{~m}$ height). Each channel had 40 pores $(6 \mathrm{~cm}$ diameter). Every unit was provided by an electric pump representing seven groups (Table, 1) nutrient solution $\left(2.0 \mathrm{dSm}^{-1}\right.$ as a control), $2000 \mathrm{mg} / \mathrm{L} \mathrm{NaCl}\left(5.42 \mathrm{dSm}^{-1}\right), 4000 \mathrm{mg} / \mathrm{L} \mathrm{NaCl}^{-1}\left(8.42 \mathrm{dSm}{ }^{-1}\right)$, $2000 \mathrm{mg} / \mathrm{L} \mathrm{CaCl}_{2}\left(4.59 \mathrm{dSm}^{-1}\right), 4000 \mathrm{mg} / \mathrm{L} \mathrm{CaCl}_{2}\left(7.60 \mathrm{dSm}^{-1}\right), 2000 \mathrm{mg} / \mathrm{L} \mathrm{NaCl}_{+} \mathrm{CaCl}_{2}(1: 1)\left(5.08 \mathrm{dSm}{ }^{-1}\right)$ and $4000 \mathrm{mg} / \mathrm{L}$ $\mathrm{NaCl}+\mathrm{CaCl}_{2}(1: 1)\left(8.08 \mathrm{dSm}^{-1}\right)$.

The seedlings were transplanted to the experimental installation on Feb, 26,2008 (after 45 days from pre-soaking) at the stage of four/five true leaves. Two uniform seedlings were transplanted to $6 \mathrm{~cm}$ perforated pots (reticulated) containing peat moss and perlite (1:1) as a rooting medium.

Every two channels was divided into 9 sets, the first set was soaked in distilled water (control), AsA, $\alpha$-tocopherol at (50 or $100 \mathrm{mg} / \mathrm{L}$ ) each, SA at (75 or $150 \mathrm{mg} / \mathrm{L})$, and Yeast extract at (1000 or $2000 \mathrm{mg} / \mathrm{L}$ ). Each set contained (8 replicates) 16 seedlings (two seedling/pot) spaced $10 \mathrm{~cm}$ representing a Nutrient Film Technique (NFT).

To keep the concentrations of sodium chloride and mineral nutrients constant, the solution was changed every 7 to 10 days and the volume of the solution was maintained by adding distilled water as required after measuring the electrical conductivity by digital conductivity meter Lutron CD-4301. A nutrient solution was pumped into the channels at a flow rate of one liter per minute from a reservoir containing 10 liters.

\section{Sampling dates:}

\section{Yield and its components:}

Harvesting was performed weekly throughout the cropping period, starting after 80 DAT and terminating after 160 DAT. At each harvest:

- $\quad$ Fruits number per plant and fruits fresh weight $(\mathrm{kg} / \mathrm{plant})$ first 4 th pickings and total yield.

- Ascorbic acid and total soluble carbohydrates (TSC) according to (Sadasivam and Manickam, 1996), total soluble solids (TSS \%) estimated by hand refractometer according to Cox and Pearson (1962). 


\section{Statistical analysis:}

The obtained data were subjected to statistical analysis of variance according to Gomez and Gomez (1984).

\section{RESULTS AND DISCUSSION}

\section{Fruit yield}

Data presented in Tables (4-5), in general, indicated that the number of fruits and fruits fresh weight per plant were significantly increased under low salinity level of $\mathrm{NaCl}+\mathrm{CaCl} 2$ in the first 4th pickings. However, $\mathrm{CaCl} 2$ salinity at $2000 \mathrm{mg} / \mathrm{L}$ led to a decrease in this respect. While, plants grown under $\mathrm{NaCl}$ salinity did not produced fruit yield in the first 4th picking. Increasing salinity levels caused a significant decrease in this respect. Moreover, the great reduction was occurred under $\mathrm{NaCl}$ followed by $\mathrm{NaCl}+\mathrm{CaCl} 2$ and $\mathrm{CaCl} 2$ at $4000 \mathrm{mg} / \mathrm{L}$ as compared to the untreated plants.

Table (4) Effect of pre-soaking seeds in SA, AsA, $\alpha$-tocopherol or Yeast extract on fruits number per plant and fruits fresh weight $\mathbf{k g} /$ plant (first $4^{\text {th }}$ pickings) of sweet pepper grown under non-saline and saline conditions using NFT.

\begin{tabular}{|c|c|c|c|c|c|c|c|c|c|c|c|c|}
\hline \multirow{3}{*}{\multicolumn{2}{|c|}{$\begin{array}{l}\text { Salinity } \\
(\mathrm{A})\end{array}$}} & \multirow[b]{3}{*}{ N.S. } & \multicolumn{3}{|c|}{ N.S.+ NaCl } & \multicolumn{3}{|c|}{ N.S. $+\mathrm{CaCl}_{2}$} & \multicolumn{3}{|c|}{$\begin{array}{c}\text { N.S.+ }\left(\mathrm{NaCl}+\mathrm{CaCl}_{2}\right) \\
(1: 1) \mathrm{w} / \mathrm{w}\end{array}$} & \multirow{3}{*}{$\begin{array}{c}\text { Mean } \\
(\mathrm{C})\end{array}$} \\
\hline & & & \multicolumn{2}{|c|}{ Conc. (B) } & \multirow{2}{*}{$\begin{array}{l}\text { Mean } \\
\left(A^{*} C\right)\end{array}$} & \multicolumn{2}{|c|}{ Conc. (B) } & \multirow{2}{*}{$\begin{array}{l}\text { Mean } \\
\left(A^{*} C\right)\end{array}$} & \multicolumn{2}{|c|}{ Conc. (B) } & \multirow{2}{*}{$\begin{array}{l}\text { Mean } \\
\left(A^{*} C\right)\end{array}$} & \\
\hline & & & 2000 & 4000 & & 2000 & 4000 & & 2000 & 4000 & & \\
\hline \multicolumn{13}{|c|}{ Fruits number per plant } \\
\hline \multicolumn{2}{|c|}{ Water } & 1.67 & 0.00 & 0.00 & 0.56 & 2.33 & 1.00 & 1.67 & 2.33 & 1.33 & 1.78 & 1.33 \\
\hline \multicolumn{2}{|c|}{ SA 75} & 2.67 & 1.67 & 0.00 & 1.44 & 3.67 & 2.67 & 3.00 & 5.00 & 2.67 & 3.44 & 2.63 \\
\hline \multicolumn{2}{|c|}{ SA 150} & 1.67 & 1.00 & 0.00 & 0.89 & 3.00 & 2.33 & 2.33 & 4.67 & 2.33 & 2.89 & 2.04 \\
\hline \multicolumn{2}{|c|}{ AsA 50} & 2.67 & 1.67 & 0.00 & 1.44 & 2.67 & 1.67 & 2.33 & 3.67 & 1.67 & 2.67 & 2.15 \\
\hline \multicolumn{2}{|c|}{ AsA 100} & 1.00 & 1.33 & 0.00 & 0.78 & 2.33 & 1.33 & 1.56 & 2.67 & 1.67 & 1.78 & 1.37 \\
\hline \multicolumn{2}{|c|}{ a-toco 50} & 2.00 & 1.67 & 0.00 & 1.22 & 2.33 & 1.33 & 1.89 & 2.67 & 2.33 & 2.33 & 1.81 \\
\hline \multicolumn{2}{|c|}{ a-toco 100} & 2.33 & 2.00 & 0.00 & 1.44 & 3.33 & 2.33 & 2.67 & 3.33 & 2.33 & 2.67 & 2.26 \\
\hline \multicolumn{2}{|c|}{ Yeast 1000} & 1.67 & 0.00 & 0.00 & 0.56 & 2.33 & 1.33 & 1.78 & 2.67 & 1.67 & 2.00 & 1.44 \\
\hline \multicolumn{2}{|c|}{ Yeast 2000} & 2.67 & 1.67 & 0.00 & 1.44 & 2.67 & 1.67 & 2.33 & 3.33 & 2.00 & 2.67 & 2.15 \\
\hline \multirow{3}{*}{$\stackrel{\substack{\mathbb{ల} \\
\Sigma}}{\Sigma}$} & $A$ & \multirow{3}{*}{2.04} & \multicolumn{2}{|l|}{1.09} & & \multicolumn{2}{|l|}{2.17} & & 2.47 & & & \\
\hline & $B$ & & 2.44 & 1.25 & & & & & & & & \\
\hline & $A^{*} B$ & & 1.22 & 0.00 & & 2.74 & 1.74 & 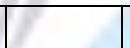 & 3.37 & 2.00 & & \\
\hline LSL & 0.05 & $A ; 0.1$ & $\mathrm{~B}$; & & C; 0.29 & $\mathrm{~A}^{*} \mathrm{~B} ; \mathrm{C}$ & 29 & $A^{*} C ; 0.5 C$ & & $; 0.50$ & $A^{*} B^{*} C$ & ;0.87 \\
\hline & & & & & ruits fre & I ve & (kg) & lant) & & & & \\
\hline $\mathrm{Wa}$ & & 0.23 & 0.00 & 0.00 & 0.08 & 0.35 & 0.10 & 0.23 & 0.33 & 0.11 & 0.22 & 0.17 \\
\hline$\overline{S A}$ & & 0.40 & 0.16 & 0.00 & 0.19 & 0.55 & 0.35 & 0.43 & 0.74 & 0.23 & 0.46 & 0.36 \\
\hline SA & & 0.24 & 0.09 & 0.00 & 0.11 & 0.46 & 0.27 & 0.32 & 0.59 & 0.21 & 0.35 & 0.26 \\
\hline AsA & & 0.34 & 0.15 & 0.00 & 0.16 & 0.44 & 0.20 & 0.33 & 0.53 & 0.14 & 0.34 & 0.28 \\
\hline AsA & & 0.17 & 0.11 & 0.00 & 0.09 & 0.29 & 0.17 & 0.21 & 0.41 & 0.13 & 0.24 & 0.18 \\
\hline a-to & & 0.30 & 0.16 & 0.00 & 0.15 & 0.34 & 0.15 & 0.26 & 0.39 & 0.18 & 0.29 & 0.24 \\
\hline$\alpha$-to & 100 & 0.33 & 0.18 & 0.00 & 0.17 & 0.51 & 0.26 & 0.37 & 0.49 & 0.20 & 0.34 & 0.29 \\
\hline Yea & 1000 & 0.22 & 0.00 & 0.00 & 0.07 & 0.37 & 0.20 & 0.26 & 0.39 & 0.18 & 0.26 & 0.20 \\
\hline Yea & 2000 & 0.36 & 0.14 & 0.00 & 0.17 & 0.41 & 0.21 & 0.33 & 0.49 & 0.17 & 0.34 & 0.28 \\
\hline & $A$ & & 0.13 & & & 0.30 & & & 0.31 & & & \\
\hline త్ర్ల & $B$ & 0.29 & 0.34 & 0.13 & & & & & & & & \\
\hline 2 & $A^{*} B$ & & 0.11 & 0.00 & & 0.41 & 0.21 & & 0.48 & 0.17 & & \\
\hline LSL & 0.05 & $A ; 0.0$ & $\mathrm{~B}$; & .02 & $C ; 0.04$ & $A^{*} B ;$ & & $A^{*} C ; 0.07$ & & ; 0.07 & $A^{*} B^{*} C$ & 0.11 \\
\hline
\end{tabular}




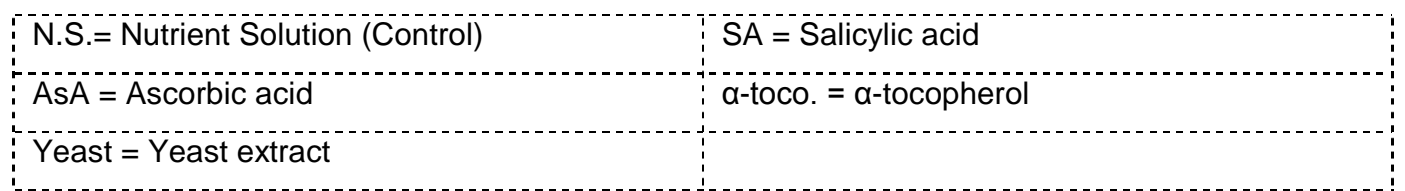

Table (5) Effect of pre-soaking seeds in SA, AsA, $\alpha$-tocopherol or Yeast extract on fruits number per plant and fruits fresh weight kg/plant (total yield) of sweet pepper grown under non-saline and saline conditions using NFT.

\begin{tabular}{|c|c|c|c|c|c|c|c|c|c|c|c|}
\hline \multirow{3}{*}{$\begin{array}{l}\text { Salinity } \\
\quad(\mathrm{A})\end{array}$} & \multirow[b]{3}{*}{ N.S. } & \multicolumn{3}{|c|}{ N.S.+ $\mathrm{NaCl}$} & \multicolumn{3}{|c|}{ N.S. $+\mathrm{CaCl}_{2}$} & \multicolumn{3}{|c|}{$\begin{array}{c}\text { N.S.+ }\left(\mathrm{NaCl}+\mathrm{CaCl}_{2}\right) \\
(1: 1) \mathrm{w} / \mathrm{w}\end{array}$} & \multirow{3}{*}{$\begin{array}{l}\text { Mean } \\
\text { (C) }\end{array}$} \\
\hline & & \multicolumn{2}{|c|}{ Conc. (B) } & \multirow{2}{*}{$\begin{array}{l}\text { Mean } \\
\left(A^{*} C\right)\end{array}$} & \multicolumn{2}{|c|}{ Conc. (B) } & \multirow{2}{*}{$\begin{array}{l}\text { Mean } \\
\left(A^{*} C\right)\end{array}$} & \multicolumn{2}{|c|}{ Conc. (B) } & \multirow{2}{*}{$\begin{array}{l}\text { Mean } \\
\left(A^{\star} C\right)\end{array}$} & \\
\hline & & 2000 & 4000 & & 2000 & 4000 & & 2000 & 4000 & & \\
\hline \multicolumn{12}{|c|}{ Fruits number per plant } \\
\hline Water & 20.00 & 17.00 & 12.00 & 16.33 & 22.33 & 16.33 & 19.56 & 22.67 & 14.33 & 19.00 & 18.30 \\
\hline SA 75 & 24.67 & 19.33 & 14.67 & 19.56 & 24.67 & 19.67 & 23.00 & 25.67 & 17.67 & 22.67 & 21.74 \\
\hline SA 150 & 23.67 & 18.33 & 14.00 & 18.67 & 23.00 & 18.67 & 21.78 & 24.67 & 16.67 & 21.67 & 20.70 \\
\hline AsA 50 & 23.67 & 18.33 & 13.33 & 18.44 & 21.67 & 19.33 & 21.56 & 24.67 & 17.33 & 21.89 & 20.63 \\
\hline AsA 100 & 20.33 & 18.00 & 13.00 & 17.11 & 21.00 & 19.00 & 20.11 & 23.67 & 17.00 & 20.33 & 19.19 \\
\hline a-toco 50 & 23.00 & 18.33 & 13.33 & 18.22 & 21.00 & 18.67 & 20.89 & 24.00 & 16.67 & 21.22 & 20.11 \\
\hline a-toco 100 & 24.33 & 18.00 & 13.33 & 18.56 & 21.33 & 19.33 & 21.67 & 24.33 & 17.33 & 22.00 & 20.74 \\
\hline Yeast 1000 & 21.67 & 18.00 & 12.67 & 17.44 & 20.67 & 17.00 & 19.78 & 22.67 & 15.00 & 19.78 & 19.00 \\
\hline Yeast 2000 & 22.67 & 18.67 & 13.67 & 18.33 & 21.33 & 17.67 & 20.56 & 24.00 & 15.67 & 20.78 & 19.89 \\
\hline \multirow{3}{*}{$\begin{array}{l}\frac{5}{\mathbb{ల}} \\
\sum \\
\sum\end{array}$} & $A$ & \multirow{3}{*}{22.67} & \multicolumn{2}{|l|}{18.07} & & \multicolumn{2}{|l|}{20.99} & & \multicolumn{2}{|l|}{21.04} & \\
\hline & $\mathrm{B}$ & & 21.38 & 16.05 & & & & & & & \\
\hline & $A^{*} B$ & & 18.22 & 13.33 & & 21.89 & 18.41 & & 24.04 & 16.41 & \\
\hline LSD at 0.05 & $\begin{array}{l}\mathrm{A} ; \\
0.25\end{array}$ & $\begin{array}{l}\mathrm{B} ; \\
0.25\end{array}$ & \multicolumn{2}{|c|}{ C; 0.43} & $\begin{array}{l}A^{*} B \\
0.43\end{array}$ & \multicolumn{2}{|c|}{$A^{*} C ; 0.75$} & \multicolumn{2}{|c|}{$\mathrm{B}^{\star} \mathrm{C} ; 0.75$} & \multicolumn{2}{|c|}{$A^{*} B^{*} C ; 1.30$} \\
\hline \multicolumn{12}{|c|}{ Total yield (kg/plant) } \\
\hline Water & 2.76 & 1.30 & 0.80 & 1.62 & 2.92 & 1.83 & 2.50 & 3.09 & 1.13 & 2.33 & 2.15 \\
\hline SA 75 & 3.43 & 1.88 & 1.25 & 2.19 & 3.56 & 2.02 & 3.01 & 3.73 & 1.48 & 2.88 & 2.70 \\
\hline SA 150 & 3.60 & 1.76 & 1.21 & 2.19 & 3.44 & 2.02 & 3.02 & 3.58 & 1.47 & 2.88 & 2.69 \\
\hline AsA 50 & 3.40 & 1.68 & 1.12 & 2.07 & 3.44 & 2.00 & 2.94 & 3.52 & 1.36 & 2.76 & 2.59 \\
\hline AsA 100 & 3.53 & 1.46 & 1.08 & 2.02 & 2.54 & 1.95 & 2.67 & 3.50 & 1.30 & 2.78 & 2.49 \\
\hline a-toco 50 & 3.19 & 1.59 & 1.11 & 1.97 & 2.88 & 1.87 & 2.65 & 3.51 & 1.26 & 2.65 & 2.42 \\
\hline a-toco 100 & 3.43 & 1.52 & 1.10 & 2.01 & 3.24 & 2.12 & 2.93 & 3.39 & 1.40 & 2.74 & 2.56 \\
\hline Yeast 1000 & 2.95 & 1.33 & 0.81 & 1.70 & 3.15 & 1.87 & 2.66 & 3.21 & 1.12 & 2.43 & 2.26 \\
\hline Yeast 2000 & 3.25 & 1.46 & 0.92 & 1.88 & 3.19 & 1.90 & 2.78 & 3.47 & 1.23 & 2.65 & 2.44 \\
\hline & $A$ & & 1.96 & & & 2.80 & & & 2.68 & & \\
\hline $\begin{array}{c}\text { స్ } \\
\mathbb{0}\end{array}$ & $B$ & 3.28 & 2.72 & 1.44 & & & & & & & \\
\hline$z$ & $A^{*} B$ & & 1.55 & 1.04 & & 3.15 & 1.95 & & 3.44 & 1.31 & \\
\hline LSD at 0.05 & $\mathrm{~A} ; 0.03$ & $\mathrm{~B} ; 0.03$ & $\mathrm{C} ; 0.05$ & & $\mathrm{~A}^{*} \mathrm{~B} ; 0.05$ & $\mathrm{~A}^{*} \mathrm{C} ; 0.09$ & & $\mathrm{~B}^{*} \mathrm{C} ; 0.09$ & & $\mathrm{~A}^{*} \mathrm{~B}^{*} \mathrm{C} ; 0$ & \\
\hline
\end{tabular}

\begin{tabular}{|c|c|}
\hline N.S.= Nutrient Solution (Control) & SA = Salicylic acid \\
\hline $\mathrm{AsA}=\mathrm{Ascorbic}$ acid & $\alpha$-toco. $=\alpha$-tocopherol \\
\hline
\end{tabular}


Pre-soaking seeds in the selected bio-stimulants used at both levels caused a significant increase in the number of sweet pepper fruits and its fresh weight per plant (first 4th pickings and total yield) under non-saline conditions. In addition, presoaking seeds in salicylic acid at $75 \mathrm{mg} / \mathrm{L}$ or ascorbic acid at $50 \mathrm{mg} / \mathrm{L}$ was more effective in this respect.

Regarding the interactions, between salinity and the selected bio-stimulants used $\left(A^{*} C\right)$, data in the same tables show that there is a significant increase in fruits number and its fresh weight per plant (first 4th pickings and total yield) as compared to untreated plants under salinity conditions. In addition, means data of $\left(A^{*} B\right)$ indicated that the fruit number and its fresh weight per plant (first 4th pickings) were significantly increased under low level of $\mathrm{CaCl} 2 \mathrm{and} \mathrm{NaCl}+\mathrm{CaCl} 2, \mathrm{but}$ plants grown under $\mathrm{NaCl}$ salinity did not produced yield in the first 4th pickings. Meanwhile, under high salinity level, application of these chemicals, in most cases, counteracted the harmful effect of salinity on growth especially salicylic acid at $75 \mathrm{mg} / \mathrm{L}$ and ascorbic acid at $50 \mathrm{mg} / \mathrm{L}$ as compared to the untreated plants under such salinity conditions.

\section{Fruit quality}

\section{Ascorbic acid and total carbohydrates concentrations in the fruit}

Data presented in Table (6) clearly show that increasing salinity levels from 2000 to $4000 \mathrm{mg} / \mathrm{L}$ of all salinity types ( $\mathrm{NaCl}, \mathrm{CaCl} 2$ and its combination 1:1) significantly increased ascorbic acid as well as total carbohydrates concentrations in sweet pepper fruits. The highest value was obtained under high salinity level in all salinity types as compared to the untreated plants. In addition, sweet pepper plants growing under $\mathrm{NaCl}$ resulted a greater increase in ascorbic acid and total carbohydrates concentrations followed by $\mathrm{NaCl}+\mathrm{CaCl} 2$ and $\mathrm{CaCl} 2$ as compared to the unstressed plants (control). Furthermore, pre-soaking seeds in SA, AsA, a-tocopherol and yeast extract at both levels increased ascorbic acid and total carbohydrates concentrations under non-saline conditions.

Moreover, AsA at both levels or SA at $75 \mathrm{mg} / \mathrm{L}$ was more effective as compared with the other treatments Regarding the interactions $\left(A^{*} B\right)$ the data in the same table show that ascorbic acid as well as total carbohydrates concentrations were significantly increased with increasing salinity level from 2000 to $4000 \mathrm{mg} / \mathrm{L}$ in all salinity types. The maximum increase was recorded for plants grown under $\mathrm{NaCl}$ followed by $\mathrm{NaCl}+\mathrm{CaCl} 2$ and $\mathrm{CaCl} 2$.

Generally, it could be concluded that, all applied bio-stimulants used enhanced ascorbic acid and total soluble carbohydrates concentrations under high salinity levels. Furthermore, AsA at both levels or SA at $75 \mathrm{mg} / \mathrm{L}$ was more effective in this respect.

\section{Total soluble solids percentage (TSS \%):}

The data presented in Table (7) clearly show that low salinity level $(2000 \mathrm{mg} / \mathrm{L})$ of all salinity types $\mathrm{NaCl}, \mathrm{CaCl} 2$ and its combination (1:1) caused a high significant increase in the TSS \%, thereafter decreased with increasing salinity level to $4000 \mathrm{mg} / \mathrm{L}$. In addition, the great reduction in TSS \% was occurred under $\mathrm{NaCl}$ at high salinity levels as compared with other salinity types. Moreover, pre-soaking seeds in SA, AsA, $\alpha$-tocopherol and yeast extract at both levels increased TSS $\%$ under non-saline conditions. Furthermore, SA at $75 \mathrm{mg} / \mathrm{L}$ or AsA at $50 \mathrm{mg} / \mathrm{L}$ were more effective as compared with the other treatments. Regarding the interactions $\left(A^{*} B\right)$ the data revealed that TSS \% was significantly increased under low salinity level $(2000 \mathrm{mg} / \mathrm{L})$ and the maximum increase was recorded for plants grown under $\mathrm{NaCl}+\mathrm{CaCl} 2$ followed by $\mathrm{CaCl} 2$ and $\mathrm{NaCl}$. While, increasing salinity level to $4000 \mathrm{mg} / \mathrm{L}$ led to a significant decrease in this respect especially under $\mathrm{NaCl}$. Generally, it could be concluded that, all chemicals used enhanced TSS \% under high salinity levels. Furthermore, AsA at $50 \mathrm{mg} / \mathrm{L}$ as well as SA at $75 \mathrm{mg} / \mathrm{L}$ were more effective in this respect. Generally, increasing salinity levels was associated with a reduction in sweet pepper yield.

This reduction in fruit yield may be attributed to a reduction in number of fruits per plant as well as fresh weight per plant (Tables, 4-5) and may be due to low production, expansion, senescence and physiologically less active green foliage (Kumar et al., 1994 and Wahid et al., 1997) and/or to a reduction in fruit set which may be attributed to a decrease in the viability of pollen grains and/or in the receptivity of the stigmatic surface (Khatun and Flowers, 1995) and/or decreasing water potential resulted from increasing salinity stress specially during microsporogenesis causing a significant increase in pollen sterility due to increasing ABA concentration (Saini and Westgate, 2000).

Table (6) Effect of pre-soaking seeds in SA, AsA, a-tocopherol or Yeast extract on concentration of ascorbic acid $(\mathbf{m g} / \mathbf{1 0 0 g} \mathrm{FW})$ and total carbohydrates ( $\mathbf{m g} / \mathrm{g}$ DW) in sweet pepper fruits grown under non-saline and saline conditions using NFT.

\begin{tabular}{|c|c|c|c|c|c|c|c|c|c|c|c|}
\hline \multirow{3}{*}{$\begin{array}{l}\begin{array}{c}\text { Salinity } \\
(\mathrm{A})\end{array} \\
\text { Treatment } \\
\text { (C) } \mathrm{mg} / \mathrm{L}\end{array}$} & \multirow[b]{3}{*}{ N.S. } & \multicolumn{3}{|c|}{ N.S.+ NaCl } & \multicolumn{3}{|c|}{ N.S. $+\mathrm{CaCl}_{2}$} & \multicolumn{3}{|c|}{$\begin{array}{c}\text { N.S. }+\left(\mathrm{NaCl}+\mathrm{CaCl}_{2}\right) \\
(1: 1) \mathrm{w} / \mathrm{w}\end{array}$} & \multirow{3}{*}{$\begin{array}{l}\text { Mean } \\
\text { (C) }\end{array}$} \\
\hline & & \multicolumn{2}{|c|}{ Conc. (B) } & \multirow{2}{*}{$\begin{array}{l}\text { Mean } \\
\left(A^{*} C\right)\end{array}$} & \multicolumn{2}{|c|}{ Conc. (B) } & \multirow{2}{*}{$\begin{array}{l}\text { Mean } \\
\left(A^{*} C\right)\end{array}$} & \multicolumn{2}{|c|}{ Conc. (B) } & \multirow{2}{*}{$\begin{array}{l}\text { Mean } \\
\left(A^{*} C\right)\end{array}$} & \\
\hline & & 2000 & 4000 & & 2000 & 4000 & & 2000 & 4000 & & \\
\hline \multicolumn{12}{|c|}{ Ascorbic acid } \\
\hline Water & 16.31 & 29.43 & 38.88 & 28.21 & 22.66 & 33.31 & 24.09 & 24.93 & 36.30 & 25.85 & 26.05 \\
\hline SA 75 & 21.15 & 31.57 & 42.37 & 31.70 & 23.90 & 34.67 & 26.57 & 27.19 & 38.37 & 28.90 & 29.06 \\
\hline
\end{tabular}




\begin{tabular}{|c|c|c|c|c|c|c|c|c|c|c|c|c|}
\hline \multicolumn{2}{|c|}{ SA 150} & 20.98 & 32.18 & 43.19 & 32.12 & 23.77 & 35.06 & 26.60 & 27.69 & 37.82 & 28.83 & 29.18 \\
\hline \multicolumn{2}{|c|}{ AsA 50} & 22.02 & 32.78 & 44.41 & 33.07 & 24.23 & 35.82 & 27.35 & 28.02 & 38.27 & 29.44 & 29.95 \\
\hline \multicolumn{2}{|c|}{ AsA 100} & 22.47 & 33.04 & 48.02 & 34.51 & 24.43 & 36.09 & 27.66 & 28.31 & 38.50 & 29.76 & 30.64 \\
\hline \multicolumn{2}{|c|}{ a-toco 50} & 21.40 & 31.77 & 42.73 & 31.97 & 24.06 & 34.92 & 26.79 & 27.35 & 37.65 & 28.80 & 29.18 \\
\hline \multicolumn{2}{|c|}{ a-toco 100} & 21.69 & 32.59 & 44.08 & 32.79 & 24.15 & 35.36 & 27.07 & 27.77 & 38.08 & 29.18 & 29.68 \\
\hline \multicolumn{2}{|c|}{ Yeast 1000} & 18.93 & 29.17 & 39.48 & 29.19 & 22.77 & 33.64 & 25.12 & 25.89 & 36.55 & 27.12 & 27.14 \\
\hline \multicolumn{2}{|c|}{ Yeast 2000} & 19.90 & 30.48 & 40.80 & 30.39 & 23.50 & 34.41 & 25.94 & 26.76 & 37.46 & 28.04 & 28.12 \\
\hline \multirow{3}{*}{$\frac{\mathbb{N}}{\stackrel{\mathbb{N}}{\mathbb{N}}}$} & $A$ & \multirow{3}{*}{20.54} & \multicolumn{3}{|l|}{31.55} & \multicolumn{3}{|l|}{26.35} & \multicolumn{3}{|l|}{28.44} & \\
\hline & B & & \multicolumn{3}{|c|}{\begin{tabular}{|l|l|}
27.42 & 38.38 \\
\end{tabular}} & & & & & & & \\
\hline & $A^{*} B$ & & 31.45 & 42.66 & & 23.72 & \multicolumn{2}{|l|}{34.81} & \multirow{2}{*}{\multicolumn{2}{|c|}{27.10}} & & \\
\hline \multicolumn{2}{|c|}{ LSD at 0.05} & \multirow[t]{2}{*}{$A ; 0.18$} & \multicolumn{2}{|c|}{$B ; 0.18$} & $C ; 0.31$ & \multicolumn{2}{|c|}{$A^{*} B ; 0.31$} & $A^{*} C ; 0.53$ & & & \multicolumn{2}{|c|}{$A^{*} B^{*} C ; 0.92$} \\
\hline \multicolumn{12}{|c|}{ Total soluble carbohydrates } & \\
\hline \multicolumn{2}{|c|}{ Water } & 45.52 & 67.31 & 80.45 & 64.43 & 58.80 & 70.89 & 58.41 & 63.96 & 74.53 & 61.34 & 61.39 \\
\hline \multicolumn{2}{|c|}{ SA 75} & 58.67 & 70.78 & 113.05 & 80.83 & 63.66 & 74.34 & 65.56 & 66.91 & 80.01 & 68.53 & 71.64 \\
\hline$\overline{S A}$ & & 55.71 & 69.76 & 84.88 & 70.12 & 61.88 & 72.79 & 63.46 & 65.55 & 76.84 & 66.03 & 66.54 \\
\hline As & & 58.41 & 70.54 & 98.98 & 75.97 & 63.39 & 73.83 & 65.21 & 66.58 & 79.31 & 68.10 & 69.76 \\
\hline As & & 57.30 & 70.11 & 90.05 & 72.49 & 62.76 & 73.24 & 64.43 & 66.17 & 77.86 & 67.11 & 68.01 \\
\hline$\alpha-t$ & 50 & 56.42 & 69.91 & 87.15 & 71.16 & 62.37 & 73.07 & 63.95 & 65.86 & 77.41 & 66.56 & 67.22 \\
\hline$\alpha-t$ & 100 & 57.91 & 70.27 & 91.47 & 73.21 & 63.17 & 73.47 & 64.85 & 66.44 & 78.58 & 67.64 & 68.57 \\
\hline $\mathrm{Ye}$ & 1000 & 51.85 & 68.52 & 81.32 & 67.23 & 59.65 & 71.21 & 60.90 & 64.10 & 74.89 & 63.61 & 63.92 \\
\hline $\mathrm{Ye}$ & 2000 & 54.10 & 69.00 & 82.95 & 68.68 & 60.75 & 72.03 & 62.29 & 64.96 & 75.90 & 64.99 & 65.32 \\
\hline & $A$ & & 71.57 & & & 63.23 & & & 65.99 & & & \\
\hline$\overline{\mathbb{O}}$ & $B$ & 55.10 & 65.67 & 80.02 & & & & & & & & \\
\hline$z$ & $A^{*} B$ & & 69.58 & 90.03 & & 61.83 & 72.76 & & 65.61 & 77.26 & & \\
\hline & 0.05 & $A ; 0.27$ & B; & & ; 0.48 & $A^{*} B ; 0$ & & $A^{*} C ; 0.82$ & $B^{*}$ & 0.82 & $\begin{array}{l}\mathrm{A}^{*} \\
1 .\end{array}$ & \\
\hline
\end{tabular}

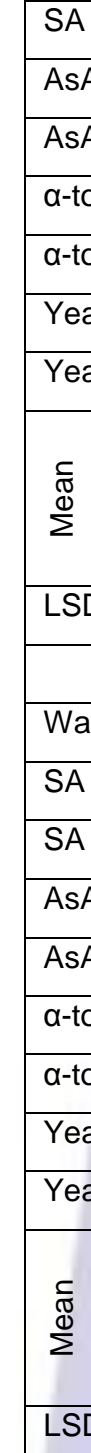

SA = Salicylic acid

N.S. = Nutrient Solution (Control)

a-toco. = a-tocophero

ÁsA = Áscorbic acid

Yeast = Yeast extract

Table (7) Effect of pre-soaking seeds in SA, AsA, a-tocopherol or Yeast extract on total soluble solids (TSS) \% in sweet pepper fruits grown under non-saline and saline conditions using NFT.

\begin{tabular}{|c|c|c|c|c|c|c|c|c|c|c|c|}
\hline \multirow{3}{*}{$\begin{array}{l}\quad \begin{array}{c}\text { Salinity } \\
\text { (A) }\end{array} \\
\text { Treatment } \\
\text { (C) } \mathrm{mg} / \mathrm{L}\end{array}$} & \multirow[b]{3}{*}{ N.S. } & \multicolumn{3}{|c|}{ N.S.+ $\mathrm{NaCl}$} & \multicolumn{3}{|c|}{ N.S.+ $\mathrm{CaCl}_{2}$} & \multicolumn{3}{|c|}{$\begin{array}{c}\text { N.S.+ } \\
(1: 1) ~ \\
(1 / w / w\end{array}$} & \multirow{3}{*}{$\begin{array}{l}\text { Mean } \\
\text { (C) }\end{array}$} \\
\hline & & \multicolumn{2}{|c|}{ Conc. (B) } & \multirow{2}{*}{$\begin{array}{l}\text { Mean } \\
\left(A^{*} C\right)\end{array}$} & \multicolumn{2}{|c|}{ Conc. (B) } & \multirow{2}{*}{$\begin{array}{l}\text { Mean } \\
\left(A^{*} C\right)\end{array}$} & \multicolumn{2}{|c|}{ Conc. (B) } & \multirow{2}{*}{$\begin{array}{l}\text { Mean } \\
\left(A^{\star} C\right)\end{array}$} & \\
\hline & & 2000 & 4000 & & 2000 & 4000 & & 2000 & 4000 & & \\
\hline \multicolumn{12}{|c|}{ Total soluble solids \% } \\
\hline Water & 4.95 & 5.19 & 3.38 & 4.51 & 5.52 & 4.69 & 5.06 & 5.98 & 4.38 & 5.10 & 4.89 \\
\hline SA 75 & 5.18 & 5.51 & 4.37 & 5.02 & 5.91 & 4.95 & 5.35 & 7.75 & 4.65 & 5.86 & 5.41 \\
\hline SA 150 & 5.08 & 5.39 & 4.21 & 4.89 & 5.77 & 4.87 & 5.24 & 6.70 & 4.58 & 5.45 & 5.20 \\
\hline AsA 50 & 5.17 & 5.49 & 4.34 & 5.00 & 5.88 & 4.94 & 5.33 & 7.38 & 4.65 & 5.73 & 5.35 \\
\hline AsA 100 & 5.10 & 5.42 & 4.27 & 4.93 & 5.81 & 4.91 & 5.28 & 6.92 & 4.60 & 5.54 & 5.25 \\
\hline
\end{tabular}




\begin{tabular}{|c|c|c|c|c|c|c|c|c|c|c|c|c|}
\hline \multicolumn{2}{|c|}{$\alpha$-toco 50} & 5.09 & 5.40 & 4.25 & 4.92 & 5.79 & 4.84 & 5.24 & 6.82 & 4.60 & 5.51 & 5.22 \\
\hline \multicolumn{2}{|c|}{ a-toco 100} & 5.12 & 5.46 & 4.31 & 4.96 & 5.85 & 4.93 & 5.30 & 7.21 & 4.63 & 5.65 & 5.30 \\
\hline \multicolumn{2}{|c|}{ Yeast 1000} & 4.96 & 5.24 & 3.87 & 4.69 & 5.56 & 4.72 & 5.08 & 6.10 & 4.43 & 5.16 & 4.98 \\
\hline \multicolumn{2}{|c|}{ Yeast 2000} & 5.01 & 5.38 & 4.16 & 4.85 & 5.71 & 4.78 & 5.17 & 6.43 & 4.48 & 5.31 & 5.11 \\
\hline \multirow{3}{*}{$\begin{array}{l}\frac{c}{\mathbb{d}} \\
\sum \\
\sum\end{array}$} & $A$ & \multirow{3}{*}{5.07} & \multicolumn{3}{|l|}{4.86} & \multicolumn{3}{|l|}{5.23} & \multicolumn{3}{|l|}{5.48} & \\
\hline & B & & \multicolumn{3}{|l|}{5.98} & & & & & & & \\
\hline & $A^{*} B$ & & 5.39 & 4.13 & & 5.75 & 4.85 & & 6.81 & 4.56 & & \\
\hline \multicolumn{2}{|c|}{ LSD at 0.05} & $A ; 0.01$ & \multicolumn{2}{|c|}{$\mathrm{B} ; 0.01$} & C; 0.02 & \multicolumn{2}{|c|}{$A^{*} B ; 0.02$} & $A^{*} C ; 0$. & \multicolumn{2}{|c|}{$\mathrm{B}^{\star} \mathrm{C} ; 0.03$} & \multicolumn{2}{|c|}{$A^{*} B^{\star} C ; 0.06$} \\
\hline
\end{tabular}

\begin{tabular}{|c|c|}
\hline N.S. = Nutrient Solution (Control) & SA = Salicylic acid \\
\hline $\mathrm{AsA}=\mathrm{A}$ scorbic acid & $\alpha$-toco. $=\alpha$-tocopherol \\
\hline
\end{tabular}

Moreover, poor seed setting characterized by sterile fruits is believed to be due to a reduction in fruit number (Tables, 4 -5) referred to substantial abscission of flowers or young fruit due to ethylene induction (Bishnoi et al., 1990). This factor affecting cell division and cell expansion, such as tissue water status and the concentration of certain plant hormones, i.e. ABA is involved in the regulation of fruit set under stress (Saini and Westgate, 2000) .

In the present investigation, presoaking seeds in phytohoromnes, vitamins and yeast extract has a beneficial effect on yield of pepper grown under saline conditions by enhancing growth and nutritional status of plant (Faissal and Hassan, 2004). These results are in agreement with El-lithy and El-Greadly (2001) who pointed out that AsA gave earliness of melon flowering and yield and increased weight of total yield but the number of fruits was not affected. In addition, Faissal and Hassan (2004) reported that AsA caused promotion on number of clusters/vine, yield/vine and cluster and berry weights in grapevines. The obtained results might be attributed to the increment in the amounts of metabolites synthesized by the plant, which, in turn, accelerated plant growth and dry weight, resulting in favorable effects on flowering and fruiting as well as finally improved the total yield.

Concerning ascorbic acid and its role in increasing carbohydrates and protein, it could be concluded that ascorbic acid play a role as activator or intermediate in the formation of carbohydrates during photosynthesis. It may be the transformative product of the sugar first found in photosynthesis (Ghourab and Wahdan, 2000). Moreover, Ahmed (2001) reported that ascorbic acid enhances the biosynthesis of carbohydrates and translocation of sugars.

Salicylic acid might be increase enzyme activity as $\alpha$-amylase, nitrate reductase which accelerates the sugar translocation from the leaves to developing fruit (Sharma et al., 1986). In addition, Haroun et al. (1998) found that low dose of salicylic acid $(2.5 \mathrm{mM})$ significantly increased total carbohydrate content in seed lupine. While, higher doses $(5$ and $10 \mathrm{mM}$ ) significantly decreased total carbohydrate content in maize grains (Abdel-Wahed et al., 2006). Moreover, Amin et al. (2008) stated that enhancement effect of total carbohydrates as well as nitrogen, phosphorus and potassium contents in wheat grains was obtained by 100 or $200 \mathrm{mg} \mathrm{L}-1$ of salicylic acid.

The stimulating effect of $\alpha$-tocopherol on yield and its quality on sweet pepper may be due to the role of $\alpha$ tocopherol in preventing the propagation of lipid peroxidation by scavenging lipid peroxyl radicals in thylakoid membranes. Sattler et al. (2004) mentioned that the only well defined tocochromanol function in plants to date is the protection of seed storage lipids from oxidation during dormancy and germination.

The positive effects of applying yeast could be attributed to its active role in hydrolysis of pectic substances, vitamins, enzymes and coenzymes that are important components of the yeast. Moreover, yeast has three basic functions, i.e. carbon dioxide production, formation of alcohol, acids, esters (Martinez-Anoya et al., 1990 and Nadia, 1995) and it contains after decomposition a wide group from amino acids and vitamins (FAO, 2008). In addition, yeast contains different nutrients especially nitrogen, phosphorus and potassium as well as some common amino acids (Abou-Zaid, 1984) and/or its capability to produce ethylene followed accumulation of phytoalexin, 6-methoxyeelein (Guo and Ohta, 1994). Thus, mechanism of yeast functions may be due to one or more of yeast components or the bio-products of reactions by yeast with other bio-organism compounds.

Dealing with the various positive effects of applying active dry yeast to plants, Idso et al. (1995) reported that these effects are attributed to their own contents of different nutrients, high protein, larger amount of vitamin B and natural plant growth regulators such as cytokinins which play a role in orientation and translocation of metabolites from leaves into the reproductive organs. Moreover, it might be play a role in the synthesis of protein degradation which might lead to the improvement of yield and its quality (El-Ghamriny et al., 1999). In addition, soluble phosphate combination with cation in soil solution to form low solubility substances called phosphate fixation which improve net photosynthesis. Moreover, Naguib and Khalil (2002) found that the enhancing effect of yeast on the growth and yield of black cumin plants could be 
attributed to its great content of minerals particularly $\mathrm{N}, \mathrm{P}$ and $\mathrm{K}$ as well as certain natural hormones, beside high amount of vitamins especially $\mathrm{B}$ which plays an important role in improving growth.

\section{REFERENCES}

1. Abdel-Wahed, M.S.A.; Amin, A.A. and El-Rashad, S.M. 2006. Physiological effect of some bioregulators on vegetative growth, yield and chemical constituents of yellow maize plants. World J. Agric. Sci., 2 (2): 149-155.

2. Abou-Zaid, M. 1984. Biochemical studies on fodder yeast. Ph.D. Thesis, Fac. Agric.Cairo Univ. Egypt.

3. Ahmed, A.M. 2001. Studies for controlling malformation and improving yield and fruit quality of hindy bisinnara mangoes by using active dry yeast, ascorbic acid and sulphur. Minia J.of Agric. Res. and Dev., 21 (2): $219-233$.

4. Amin, A.A.; Rashad, El-Sh.M. and Fatma, A.E. Gharib. 2008. Changes in morphological, physiological and reproductive characters of wheat plants as affected by foliar application with salicylic acid and ascorbic acid. Aust. J. Basic and Appl. Sci., 2 (2): 252-261.

5. Bishnoi, N.R.; Laura, J.S.; Sharma, K.D.; Singh, N. and Singh, N. 1990. Effect of salinity, salinization and desalinization on flowering and various yield parameters in pea (Pisum sativum L.) and chickpea (Cicer arietinum L.). Int. J. Tropical Agric., 8 (2): 148-153.

6. Casado-Vela, J.; Sellés, S.; Díaz-Crespo, C.; Navarro-Pedreňo, J.; Mataix-Beneyto, J. and Gómez, I. 2007. Effect of composted sewage sludge application to soil on sweet pepper crop (Capsicum annuum var. annuum) grown under two exploitation regimes. Waste Manag., 27: 1509-1518.

7. Cooper, A. 1979. The ABC of NFT. Growers Books, London, pp. 59.

8. Cox, H.E. and Pearson, D.C. 1962. The chemical analysis of foods, Chemical Publishing Co. Inc., New York, pp: 136-144.El-Ghamriny,

9. E.A.; Arisha, E.A.H. and Nour, K.A. 1999. Studies on tomato flowering, fruit set, yield and quality in summer season. I. Spraying with thiamine, ascorbic acid and yeast. Zagazig J. Agric. Res., 26 (5): 1345-1364.

10. El-Lithy, Y.T. and El-Greadly, N.H. 2001. Study on the effect of ascorbic acid, nicotinamide and their combination on growth, flowering, yield, fruit quality and endogenous hormones of melon plants under effect of salinity of water irrigation. J. Agric. Sci. Mans. Univ., 26 (7): 5101-5114.

11. Etehadnia, M. 2009. Salt stress tolerance in potato genotypes. Ph.D. Thesis, College of Graduate Studies and Research, Univ. of Saskatchewan, Saskatoon.

12. Faissal, F.A. and Hassan, A.M. 2004. Influence of some antioxidants on growth, vine nutritional status, yield and quality of berries in banaty grapevines. Assiut. J. Agric. Sci., 35 (4): 131-140.

13. FAO, 2008. FAO Land and Plant Nutrition Management Service. http://www.fao.org/ag/agl/agll/spush

14. Ghourab, M.H.H. and Wahdan, G.A. 2000. Response of cotton plants to foliar application of ascorbine and ascorbic acid. Egypt. J. Agric. Res., 78 (3): 1195-1206.

15. Guo, Z.J. and Ohta, Y. 1994. Effect of ethylene biosynthesis on the accumulation of 6-methoxymellein induced by elicitors in carrot cells. J. Plant Physiol., 144 (6): 700- 704.

16. Haroun, S.A.; Al-Desuqy, H.S.; Shukry, W.M. and Gaber, A.M. 1998. Regulation of growth and metabolism in Lupinus termis plant by sodium salicylate. Egypt J. Physiol. Sci., 22.

17. Idso, S.B.; Idso, K.E. and Hoober, J.K. 1995. Effect of atmospheric CO2 enrichment and foliar methanol application on net photosynthesis of sour orange trees (Citrus aurantium) leaves. Am. J. Bot., 82 (1): 26-30.

18. Khatun, S. and Flowers, T.J. 1995. Effects of salinity on seed set in rice. Plant Cell and Environ., 18: 61-67.

19. Khosravinejad, F.; Heydari, R. and Farboodnia, T. 2009. Effect of salinity on organic solutes contents in barley. Pak. J. Biol. Sci., 12: 158-162.

20. Kumar, S.; Naidu, K.M. and Sehtia, H.L. 1994. Causes of growth reduction in elongating and expanding leaf tissue of sugarcane under saline conditions. Aust. J. Plant Physiol., 21: 79-83.

21. Lycoskoufis, I.H.; Savvas, D. and Mavrogianopoulos, G. 2005. Growth, gas exchange and nutrient status in pepper (Capsicum annuum L.) grown in recirculating nutrient solution as affected by salinity imposed to half of the root system. Scientia Hort., 106: 147-161.

22. Martinez-anoya, M.A.; Pitarch, B.; Bayarri, P. and Beneditode Barber, C. 1990. Microflora of the sourdoughs wheat flour bread interaction between yeast and lactic acid bacteria in wheat doughs and their effects on bread quality. Cereal Chem., 67 (1): 85.

23. Munns, R. 2002. Comparative physiology of salt and water stress. Plant, Cell and Environ., 25: 239-250.

24. Munns, R. and Tester, M. 2008. Mechanisms of salinity tolerance. Annu. Rev. Plant Biol., 59: 651-681.

25. Nadia, H.A.A. 1995. Biochemical studies on improving products. M.Sc. Thesis, Fac. of Agric. Cairo Univ. Egypt. 
26. Nagodawithana, W.T. 1991. Yeast technology. Universal foods cooperation Milwauke, Wisconsin. Published by Van Nostrand, New York, pp. 273.

27. Naguib, N.Y. and Khalil, M.Y. 2002. Studies on the effect of dry yeast, thiamine and biotin on the growth and chemical constituents of black cumin (Nigella sativa L.). Arab Univ. J. Agric. Sci., 10 (3): 919-937.

28. Navarro, J.M.; Flores, P.; Garrido, C. and Martinez, V. 2006. Changes in the contents of antioxidant compounds in pepper fruits at different ripening stages, as affected by salinity. Food Chem., 96: 66-73.

29. Sadasivam, S. and Manickam, A. 1996. Biochemical methods. Second Ed., New age Int., India.

30. Saini, H.S. and Westgate, M.E. 2000. Reproductive development in grain crops during drought. Adv. in Agron., 68: 59-96.

31. Sattler, S.E.; Gilliland, L.U.; Magallanes-Lundback, M.; Pollard, M. and Della-Penna, D. 2004. Vitamin E is essential for seed longevity and for preventing lipid peroxidation during germination. Plant Cell, 16:1419-1432.

32. Sharma, S.; Sharma, S.S. and Rau, V.K. 1986. Reversal by phenolic compounds of abscussic acid-induced inhibition of in vitro activity of amylase from seeds of Triticum aestivum L. New Phytol., 103 (2): 293-297.

33. Tester, M. and Davenport, R. 2003. Na+tolerance and Na+transport in higher plants. Ann. Bot., 91: 503-527.

34. Wahid, A.; Rao, R. and Rasul, E. 1997. Identification of salt tolerance traits in sugarcane lines. Field Crop. Res., 54: 9-17.

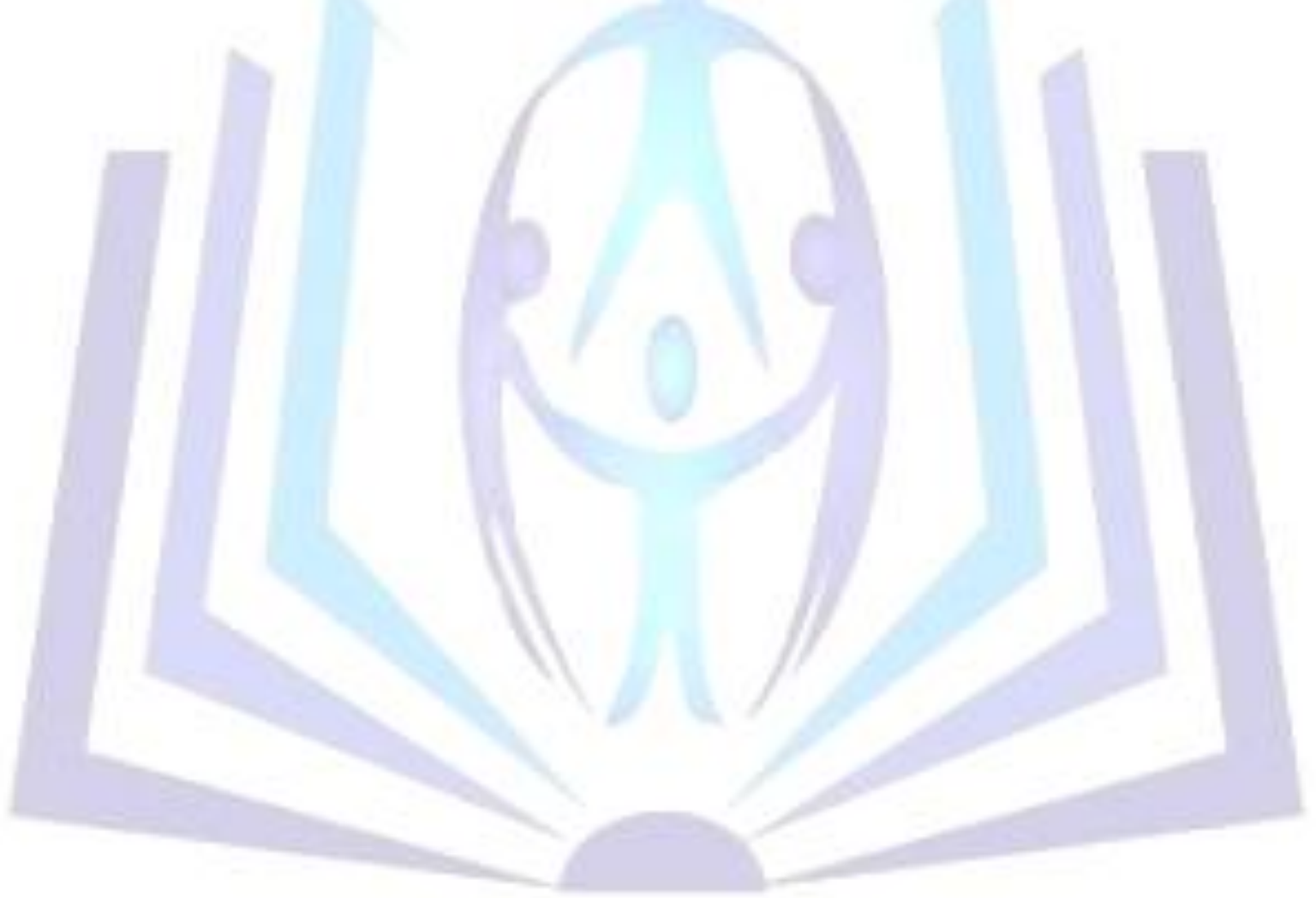

